\title{
CUIDADOS DE ENFERMAGEM MAIS VALORIZADOS E FREQUENTES DURANTE INTERNAÇÃO PSIQUIÁTRICA
}

\section{THE MORE VALUE AND FREQUENT NURSING CARE DURING PSYCHIATRIC HOSPITALIZATION}

\section{LOS CUIDADOS EN LA ENFERMERÍA MÁS VALORADOS O FRECUENTES DURANTE LA INTERNACIÓN PSIQUIÁTRICA}

\author{
Renata Marques de Oliveira ${ }^{1}$, Antonio Carlos Siqueira Júnior ${ }^{2}$, Antonia Regina Ferreira \\ Furegato $^{3}$
}

\section{RESUMO}

Objetivo: identificar os cuidados de enfermagem que são mais valorizados e aqueles que ocorrem com maior frequência durante a internação psiquiátrica, segundo a opinião de portadores de transtornos mentais, enfermeiros e técnicos de enfermagem. Métodos: estudo transversal. Participaram 27 pacientes, 6 enfermeiros e 10 técnicos enfermagem de unidade de internação psiquiátrica. Realizadas entrevistas a partir de 13 fotos de cuidados de enfermagem. Elas eram embaralhadas e entregues aos participantes para que as ordenassem segundo importância e ocorrência. Análise descritiva. Resultados:os participantes discordaram sobre os cuidados mais importantes: enfermeiros (50\%: "passagem plantão"), técnicos (40\%: "ouvir atentamente"), (37\%: "apoio espiritual"). Embora o apoio espiritual tenha sido a intervenção mais valorizada pelos pacientes, foi a menos frequente na opinião dos três grupos(76,7\%). Conclusão:o cuidado de enfermagem ocorria de modo generalista com ênfase em cuidados técnicos básicos. É preciso considerar a opinião dos usuários, pois o cuidado que eles mais valorizavam era o menos implementado.

Descritores: Cuidados de enfermagem; Unidade hospitalar de psiquiatria; Enfermagem psiquiátrica.

\footnotetext{
${ }^{1}$ Escola de Enfermagem de Ribeirão Preto. Ribeirão Preto, SP, Brasil.

${ }^{2}$ Faculdade de Medicina de Marília. Marília, SP, Brasil.

${ }^{3}$ Escola de Enfermagem de Ribeirão Preto. Ribeirão Preto, SP, Brasil.
} 


\begin{abstract}
Aim: To identify the more value and frequent nursing care in the assistance to the psychiatric patients, hospitalized in general hospital, according to the perception of mental disorder subject and nursing. Method: Cross-sectional study with 27 patients ( $\geq 18$ years), 6 nurses and 10 nurse assistants of psychiatric unity from general hospital. The nursing cares more value and frequents was investigated from 13 pictures, scrambled, the persons ordered the pictures according to their relevancy and occurrence. Descriptive analysis. Results: The participants disagreed about the most important nursing care: nurses (50\%:"shift report"), nurse assistant (40\%:"listen to the patients"), patients (37\%:"spiritual support"). Although the spiritual support was the most valued in the patients' opinion, was the less frequent in the opinion of the three groups $(76.7 \%)$. Conclusion: the nursing care occurred with an emphasis on basic technical care. It is important to consider users' opinion because the care they most valued was the less implemented.
\end{abstract}

Descriptors: Nursing care; Psychiatric unit; Psychiatric nursing.

\title{
RESUMEN
}

Objetivo: Identificar los cuidados de enfermería más valorados y frecuentes en la asistencia a pacientes psiquiátricos, hospitalizados en hospital general, según la percepción de los portadores de trastorno mental y profesionales. Método: Estudio descriptivo con 27 pacientes, 6 enfermeros y 10 auxiliares de enfermería de unidad psiquiátrica. Los cuidados más valorados y frecuentes fueron investigados a partir de 13 fotografías(los sujetos ordenaban según su importancia y ocurrencia). Análisis descriptiva. Resultados: Para $50 \%$ de los enfermeros, el cuidado más importante es el pasaje de turno; para $40 \%$ de los auxiliares de enfermería es oír al paciente y para $37 \%$ de los pacientes es el suporte espiritual. Aunque el apoyo espiritual fue la intervención más valorada por los pacientes, fue la menos frecuente. Conclusión: El cuidado de enfermería ocurría de modo generalista con énfasis en cuidados técnicos. Es necesario considerar la opinión de los usuarios, pues el cuidado que ellos más valoraban era el menos implementado.

Descriptores: Atención de enfermeira; Servicio de psiquiatría en Hospital; Enfermería psiquiátrica.

\section{INTRODUÇÃO}

A importância atribuída ao cuidado

de enfermagem, pela pessoa que o vivencia, depende do contexto cultural em que ele ocorre. Isso fica evidente ao olhar para a história do cuidado em psiquiatria que vem passando por diversas discussões, reflexões e mudanças de concepções e práticas, ao longo dos anos.

Até o século XV, os "doentes mentais" eram submetidos a intervenções mágico-religiosas. Acreditava-se que a alteração do seu comportamento era castigo divino ou possessão demoníaca. A 
partir de Pinel entendeu-se que essas pessoas eram doentes, o que favoreceu a criação dos "hospitais" que, baseados no tratamento moral, protegiam a sociedade, dando-se início à psiquiatria moderna. $\mathrm{O}$ cuidado consistia no isolamento, na repressão, na contenção e em procedimentos técnicos, alguns até agressivos, visando à manutenção da vida em seus aspectos biológicos e a contenção da agressividade que se manifesta em algumas dessas doenças. ${ }^{1}$

Ao longo do tempo, a instituição hospitalar foi transformando-se nos grandes manicômios. Este panorama sofreu importantes alterações a partir da evolução do conhecimento medicamentoso, da psicanálise e da psicologia comportamental. Em meados do século $\mathrm{XX}$, a evolução do conhecimento culminou em diversos questionamentos sobre os manicômios, reforçados pela antipsiquiatria, pela psiquiatria preventiva e pelas propostas de reversão desse processo. Organizações governamentais e não governamentais reagiram e, hoje, no Brasil, vivencia-se o movimento de reforma psiquiátrica paralelamente a um movimento mais amplo que é a reforma sanitária, propondo uma assistência humanizada e reordenando as suas consequências. $^{2}$

A enfermagem psiquiátrica, por meio das transformações em sua prática assistencial, contribuiu para a mudança da concepção de cuidado em que a pessoa é vista de modo integral, tornando-se protagonista e não mera expectadora de todo o processo. $^{2-3}$ No contexto da internação psiquiátrica, os profissionais de enfermagem são essenciais para a elaboração de um plano terapêutico em consonância com as necessidades dos pacientes e o cuidado humanizado favorece a proximidade e a melhor qualidade dos seus resultados. ${ }^{4-6}$

Com a preocupação em oferecer uma assistência de qualidade, pautada pela Lei da Reforma Psiquiátrica (10.216/2001), atualmente, estimula-se o tratamento dos portadores de transtornos mentais na rede de serviços de saúde, com ênfase nos serviços extra hospitalares, sendo a internação o último recurso. Apesar disso, estudos ainda evidenciam índices elevados de reinternações psiquiátricas. ${ }^{7-8}$ Embora sejam evidentes os avanços nas últimas décadas, o modelo biomédico ainda prevalece em muitos serviços psiquiátricos, 
influenciando as ações da enfermagem e dos demais profissionais da saúde mental.

Sendo a enfermagem a profissão mais próxima dos pacientes durante a internação, investigar como esses profissionais estão, atualmente, desempenhando suas ações no cuidado aos portadores de transtornos mentais pode fornecer informações valiosas sobre os avanços no cuidado em psiquiatria. Será que os avanços se limitam à teoria ou se concretizam, efetivamente, na prática? A obtenção dessas informações pode ajudar a refletir sobre o cuidado em psiquiatria e contribuir para aprimorar a qualidade da assistência, favorecendo a plena recuperação do portador de transtorno mental.

Hipótese: O cuidado de enfermagem na internação psiquiátrica ocorre de modo generalista, com ênfase em cuidados técnicos básicos.

Este estudo teve por objetivo identificar os cuidados de enfermagem que são mais valorizados e aqueles que ocorrem com maior frequência durante a internação psiquiátrica, segundo a opinião de portadores de transtornos mentais, enfermeiros e técnicos de enfermagem.

\section{MÉTODOS}

Estudo transversal realizado entre agosto e outubro de 2011 em uma unidade psiquiátrica de hospital geral público (UPHG) do interior de São Paulo, Brasil. A UPHG dispõe 18 leitos para internação de pessoas em surto psiquiátrico com percentual médio de ocupação de 89,0\%.

A amostra foi composta por 27 pacientes e 16 profissionais de enfermagem (seis enfermeiros e dez técnicos de enfermagem). Critérios inclusão pacientes: estar internado na UPHG entre agosto e outubro/2011. Critérios exclusão pacientes: retardo mental; < 18 anos; prejuízo da fala; recusas. Critérios inclusão profissionais: integrar a escala de enfermeiros ou de técnicos de enfermagem da UPHG entre agosto e outubro/2011. Critérios exclusão profissionais: enfermeiros ou técnicos de enfermagem de outros setores atuando temporariamente na UPHG e recusas.

Foi elaborado, especialmente para este estudo, o instrumento "Ordenação de Fotos sobre os diferentes Cuidados de Enfermagem" (OFCE). Por se tratar de um instrumento projetivo que visa obter informações indiretas (ou seja, por meio de fotos), para fins descritivos (frequência de 
ocorrência), sem pretensão de mensuração, não foi necessária sua validação. O OFCE é composto por 13 fotos que retratam diferentes ações realizadas no cuidado de enfermagem. Para a escolha dessas ações, foi consultada a Lei $\left(n^{0} 7498 / 1986\right)$ que regulamenta o exercício profissional da enfermagem no Brasil e a resolução $n^{0}$ 427/2012 do Conselho Federal de Enfermagem (Cofen).

O objetivo da enfermagem é atender as necessidades humanas básicas, transcendendo as ações rotineiras de modo a ampliar seu campo de atuação para ações terapêuticas e humanistas. Wanda Aguiar Horta fez a primeira proposta da enfermagem brasileira de processo de enfermagem com base na teoria das necessidades humanas básicas. ${ }^{9}$

De modo a incluir no OFCE fotos que retratassem os cuidados de enfermagem prestados ao paciente visto de modo holístico, definiu-se que a escolha das ações seria baseada na Classificação de João Mohana para as Necessidades Humanas Básicas: 1) psicobiológicas, 2) psicossociais e 3) psicoespirituais. $^{9}$

Embora se reconheça que, inicialmente, a teoria das necessidades humanas básicas tenha sido proposta por Maslow, optou-se por utilizar a classificação de João Mohana por ser considerada mais abrangente. Foram escolhidas, intencionalmente, quatro necessidades psicobiológicas, quatro necessidades psicossociais e uma necessidade psicoespiritual de João Mohana. A cada uma foi atribuída uma intervenção de enfermagem conforme a Classificação das intervenções de enfermagem (NIC), elaborada por pesquisadores da Universidade de Iowa ${ }^{10}$ (Figura 1).

\begin{tabular}{|l|l|l|}
\hline Teoria João Mohana & Intervenções enfermagem segundo NIC \\
\hline Necessidades & Cuidado corporal & Banho \\
\cline { 2 - 3 } psicobiológicas & Integridade física & Contenção mecânica \\
\cline { 2 - 3 } & Regulação & Controle sinais vitais \\
\cline { 2 - 3 } & Terapêutica & Administração medicamentos \\
\hline Necessidades & Atenção & Ouvir atentamente \\
\hline
\end{tabular}




\begin{tabular}{|l|l|l|}
\hline \multirow{2}{*}{ psicossociais } & Participação/gregária & Grupos de apoio \\
\cline { 2 - 3 } & Recreação & Desenvolvimento habilidades sociais \\
\cline { 2 - 3 } & Segurança & Presença/estar junto \\
\hline $\begin{array}{l}\text { Necessidade } \\
\text { psicoespiritual }\end{array}$ & Necessidades religiosas & Apoio espiritual (abordagem espiritual) \\
\hline
\end{tabular}

Figura 1 - Necessidades psicobiológicas, psicossociais e psicoespirituais, segundo a classificação de João Mohana e intervenções de enfermagem

Após definição dos nove cuidados de enfermagem relacionados às necessidades humanas básicas, consultou-se a $\mathrm{NIC}^{10}$ a fim selecionar intervenções realizadas rotineiramente pelos profissionais de enfermagem sem contato direto com os pacientes. Quatro foram escolhidas: 1) anotação, 2) desenvolvimento da equipe (capacitação), 3) passagem de plantão e 4) reunião multidisciplinar. As fotos do OFCE foram selecionadas na internet e impressas em tamanho $10 \times 8 \mathrm{~cm}$ com indicação das web páginas de onde foram obtidas. Foram plastificadas individualmente.

Realizaram-se entrevistas individuais, conduzidas por um único pesquisador, em um consultório da UPHG. Após apresentação da proposta de pesquisa e assinado o Termo de Consentimento, as 13 fotos do OFCE eram embaralhadas e entregues aos participantes solicitando que eles as colocassem sobre a mesa de acordo com a ordem de importância que atribuía a cada cuidado de enfermagem.

Em seguida, as fotos eram embaralhadas novamente e aos participantes era solicitado que as ordenassem segundo a ordem de ocorrência, ou seja, indicando quais ações de enfermagem ocorriam com maior frequência durante a internação. As ordens de importância e de ocorrência, indicadas pelo participante, eram registradas. Durante esses procedimentos, os participantes foram instruídos a relatar sua experiência com cada cuidado de enfermagem apresentado, tendo os relatos sido gravados (o conteúdo dessas gravações não é objeto de análise do presente estudo).

Além de analisar as 13 intervenções de enfermagem individualmente, utilizouse a classificação da $\mathrm{NIC}^{10}$ de cuidados direitos e indiretos. As intervenções foram assim classificadas: cuidados diretos: banho; contenção mecânica; monitoração 
sinais vitais (SSVV); administração de medicação; ouvir atentamente; grupos de apoio; desenvolvimento de habilidades sociais; presença (estar junto) e apoio espiritual (abordagem espiritual); cuidados indiretos: anotação; desenvolvimento da equipe (capacitação); passagem de plantão e reunião multidisciplinar.

As informações sobre a ordenação das 13 fotos foram tabuladas no Excel e transferidas para o Stata (versão 10.10). Foram utilizadas ferramentas de estatística descritiva (média, valor mínimo, valor máximo, frequência absoluta e frequência relativa).

$\mathrm{O}$ estudo respeitou as exigências formais contidas nas normas nacionais e internacionais regulamentadoras de pesquisas envolvendo seres humanos.

\section{RESULTADOS}

A duração média das entrevistas com os profissionais de enfermagem foi de 75,2 minutos e com os pacientes de 52,7 minutos. Quatro dos 14 técnicos de enfermagem que estavam atuando na UPHG no período da coleta dos dados não aceitaram participar do estudo. Todos os enfermeiros consentiram $(n=6)$, tendo a amostra sido composta por 16 profissionais
(10 técnicos de enfermagem e seis enfermeiros). A maioria dos profissionais de enfermagem era mulher $(68,8 \%)$ com média etária de 35,7 anos (25 a 51 anos). Para todos os enfermeiros e $40 \%$ dos técnicos, era a primeira vez que trabalhavam em psiquiatria.

Dos 84 portadores de transtornos mentais que foram internados na UPHG no período da coleta dos dados, 57 (67,9\%) não foram incluídos no estudo. Os motivos para as exclusões: recusas $(21,1 \%)$; prejuízo na fala $(17,5 \%) ;<18$ anos (26,3\%); retardo mental (5,3\%); alta hospitalar sem planejamento prévio (28\%) e reinternação $(1,8 \%)$. Dentre os 27 pacientes que participaram deste estudo, a maioria era mulher $(66,7 \%)$. Aproximadamente metade (48\%) tinha entre 20 e 39 anos de idade. A esquizofrenia foi o diagnóstico mais frequente $(44,4 \%)$.

A ordenação das 13 fotos revela que os enfermeiros, os técnicos de enfermagem e os pacientes discordam sobre os cuidados de enfermagem mais importantes durante a internação em UPHG. Metade dos enfermeiros elegeu a "passagem de plantão" o cuidado mais importante; $40 \%$ dos técnicos de enfermagem escolheram 
"ouvir atentamente" e 37\% dos pacientes “apoio espiritual". Metade dos enfermeiros e a maioria dos pacientes indicou a "contenção mecânica" como o cuidado menos importante durante a internação. Entre os técnicos de enfermagem, somente $10 \%$ manifestaram essa mesma opinião (Tabela 1).

Tabela 1 - Cuidados de enfermagem valorizados, segundo a opinião dos enfermeiros, técnicos de enfermagem e pacientes

\begin{tabular}{lccc}
\hline Cuidados de enfermagem & $\begin{array}{c}\text { Enfermeiros } \\
(\mathbf{n = 6})\end{array}$ & $\begin{array}{c}\text { Técnicos enfermagem } \\
(\mathbf{n = 1 0})\end{array}$ & $\begin{array}{c}\text { Pacientes } \\
(\mathbf{n = 2 7})\end{array}$ \\
\hline Mais importantes & $\%$ & $\%$ & $\%$ \\
Administração de medicação & - & 20,0 & 11,1 \\
Contenção mecânica & - & - & 3,7 \\
Desenvolvimento da equipe & 33,3 & - & 7,4 \\
Passagem de plantão & 50,0 & 40,0 & 7,4 \\
Ouvir atentamente & - & - & 22,2 \\
Presença/estar junto & 16,7 & - & 7,4 \\
Reunião multidisciplinar & - & 10,0 & 37,0 \\
Apoio espiritual & - & - & 3,7 \\
Verificação SSVV & - & 10,0 & 3,7 \\
Menos importantes & & 10,0 & - \\
Anotação & - & 10,0 & 55,6 \\
Banho & 33,3 & 10,0 & - \\
Contenção mecânica & 50,0 & - & 14,8 \\
Desenvolvimento equipe (capacitação) & - & - & 11,1 \\
Desenvolvimento habilidades sociais & - & - & 3,7 \\
Grupo de apoio & - & 20,0 & 7,4 \\
Passagem de plantão & - & 40,0 & 3,7 \\
Reunião multidisciplinar & - & & \\
Apoio espiritual & 16,7 & & \\
\hline & & & \\
\hline
\end{tabular}

Os enfermeiros e os técnicos de enfermagem foram coerentes quanto à escolha dos cuidados mais importantes e frequentes. Metade dos enfermeiros e dos técnicos indicou que o cuidado de enfermagem que mais realizava na UPHG 
era a "passagem de plantão" e "ouvir atentamente", respectivamente. Ao observar as escolhas dos pacientes, identifica-se que o cuidado que eles consideravam mais importante (apoio espiritual) foi apontado, por quase $90 \%$, como o menos frequente durante a internação psiquiátrica. A maioria dos pacientes (70\%) afirmou que a administração de medicação e a verificação dos sinais vitais eram os cuidados mais frequentes (Tabela 2).

Tabela 2 - Cuidados de enfermagem frequentes, segundo a opinião dos enfermeiros, técnicos de enfermagem e pacientes

\begin{tabular}{lccc}
\hline Cuidados de enfermagem & Enfermeiros (n= 6) & Técnicos $(\mathbf{n}=\mathbf{1 0})$ & Pacientes $(\mathbf{n}=\mathbf{2 7})$ \\
\hline Mais frequentes & & & \\
Administração medicação & - & 10,0 & 37,0 \\
Anotação & - & 10,0 & 7,4 \\
Desenvolvimento da equipe & - & - & 7,4 \\
Grupos de apoio & - & - & 3,7 \\
Ouvir atentamente & 33,3 & 50,0 & 3,7 \\
Passagem de plantão & 50,0 & 30,0 & 3,7 \\
Presença/estar junto & 16,7 & - & 3,7 \\
Verificação SSVV & - & - & 7,3 \\
Menos frequentes & 16,7 & 10,0 & 3,7 \\
Contenção mecânica & 16,7 & - & - \\
Desenvolvimento da equipe & 16,7 & - & - \\
Grupos de apoio & 16,7 & 20,0 & 88,9 \\
Reunião multidisciplinar & 33,3 & 70,0 & \\
Apoio espiritual & & & \\
\hline
\end{tabular}

Ao classificar os cuidados de enfermagem em diretos e indiretos, verifica-se que as opiniões dos técnicos de enfermagem e dos pacientes foram semelhantes. Setenta porcento dos técnicos e $77,8 \%$ dos pacientes elegeram os cuidados diretos como os mais importantes durante a internação em UPHG. Contrariamente, $83,3 \%$ dos enfermeiros elegeram os cuidados indiretos.
Para os técnicos de enfermagem e para os pacientes, os cuidados diretos, além de terem sido considerados mais importantes, foram também os mais frequentes $\quad(60 \%$ e $81,5 \%$, respectivamente). A indicação feita pelos enfermeiros dos cuidados que consideravam mais importantes e os que ocorriam com maior frequência, em sua prática diária, foi diferente. Enquanto $83,3 \%$ consideravam os cuidados indiretos 
mais importantes, $50 \%$ disseram que realizavam os cuidados diretos com mais frequência. No que tange as necessidades humanas básicas, dentre os participantes que consideravam os cuidados diretos mais importantes, $47,6 \%$ dos pacientes escolheram o cuidado que atendia às necessidades psicoespirituais e $57,1 \%$ dos técnicos de enfermagem o que atendia as necessidades psicossociais. $\mathrm{O}$ único enfermeiro que considerou os cuidados diretos mais importantes escolheu o cuidado que supria as necessidades.

\section{DISCUSSÃO}

Ao investigar os cuidados que os profissionais de enfermagem e os pacientes consideravam mais importantes, durante internação em UPHG, constataram-se opiniões divergentes. Dentre os 13 cuidados de enfermagem apresentados aos participantes, o apoio espiritual foi o mais valorizado pelos pacientes.

Uma pesquisa realizada a partir da consulta de 543 livros de enfermagem identificou que $32 \%$ ressaltavam a importância de avaliar as necessidades espirituais dos pacientes, porém poucos mencionavam como essa avaliação poderia ser realizada. Os autores concluíram que a escassez do tema espiritualidade nos livros didáticos indica que esse aspecto do cuidado é menos valorizado em comparação a outras intervenções de enfermagem. ${ }^{11}$

De fato, a desvalorização do apoio espiritual em relação aos demais cuidados foi percebida neste estudo. A maioria dos profissionais de enfermagem não $o$ considerou importante. Por não ser consolidado na literatura científica e nos livros didáticos, é difícil para os profissionais de enfermagem compreenderem como podem fornecer $\mathrm{o}$ apoio espiritual de modo a respeitar as necessidades individuais de cada paciente, porém sem ir além de suas habilidades, competências técnicas e profissionais.

Estudo com 139 esquizofrênicos revelou que o bem estar espiritual está associado à melhor qualidade de vida. ${ }^{12}$ Destaca-se a relevância de os profissionais avaliarem as necessidades espirituais/religiosas de cada paciente e de somente estimulá-la se houver o entendimento de que ela possa ser benéfica para o indivíduo. Entende-se que o apoio espiritual não é o enfermeiro se colocar como um religioso, mas identificar e respeitar o que é importante para o 
paciente naquele momento. A partir da escuta e do diálogo respeitoso deve-se permitir que os pacientes falem sobre suas crenças, de modo que a eles não seja negado discutir sobre aquilo que consideram importante.

Reconhece-se, todavia, que o modo como o enfermeiro aborda as necessidades espirituais dos pacientes depende do conhecimento que tem sobre o assunto, sobre si próprio e do quão à vontade se sente diante dessa temática. ${ }^{13}$ Se o que o paciente necessita ultrapassa sua competência profissional, o enfermeiro pode dar o encaminhamento necessário por meio de solicitação de visita religiosa ou de outra ação que possa confortar o paciente naquele momento.

Embora o apoio espiritual tenha sido a intervenção mais valorizada pelos pacientes, foi a menos frequentemente implementada na opinião dos três grupos de participantes (enfermeiros, técnicos e pacientes). Como o apoio espiritual não era realizado com frequência, os pacientes podem ter escolhido essa intervenção de enfermagem como a mais importante por não terem clareza do que se tratava. É possível que tenham confundido apoio espiritual com atenção, acolhimento e apoio emocional.

A discrepância entre o que é valorizado pelos pacientes e o que ocorre na assistência de enfermagem leva a questionar o cuidado que os técnicos de enfermagem elegeram como $o$ mais importante: "ouvir atentamente" o que a pessoa tem a dizer. Se o que os pacientes desejam ao referir-se ao apoio espiritual é maior atenção, isso sugere que o "ouvir atento" não tem sido utilizado de modo adequado como ferramenta terapêutica durante a internação.

Embora os técnicos de enfermagem insistam que "ouvir atentamente" é o cuidado que realizam com maior frequência, o relato dos pacientes, enquanto protagonistas do cuidado, é a fonte mais segura para identificar o que ocorre no cotidiano da UPHG. A partir das entrevistas dos pacientes, confirma-se a hipótese de que o cuidado de enfermagem, na internação psiquiátrica, se dá de modo generalista com ênfase em cuidados técnicos básicos, uma vez que mais de dois terços dos pacientes contaram que a administração de medicação e a verificação dos sinais vitais são os cuidados mais frequentes. Estudo israelense com 100 
profissionais de enfermagem de um hospital psiquiátrico mostrou que eles avaliam positivamente a utilização de psicofármacos, embora seu conhecimento sobre o assunto seja considerado moderado. $^{14}$

A opinião dos enfermeiros é diferente da opinião dos técnicos de enfermagem e dos pacientes. Para eles, os cuidados indiretos são mais importantes, especialmente a passagem de plantão. Esse resultado era esperado, tendo em vista o envolvimento desses profissionais com atividades burocráticas no seu cotidiano de trabalho. Um estudo conduzido com 13 enfermeiros, de diferentes unidades de um hospital geral brasileiro, mostrou que eles se distanciam do cuidado direto aos pacientes devido às demandas administrativas, delegando a outros profissionais, especialmente aos de nível técnico, ações que deveriam ser desempenhadas por eles próprios. Destacase a necessidade de articular as ações de cuidado direto e as gerenciais, de modo que os pacientes não sejam privados da assistência de enfermagem qualificada. ${ }^{15}$

Em relação ao cuidado menos valorizado, houve consenso entre os enfermeiros e os pacientes. A maioria elegeu a contenção mecânica, possivelmente devido à agressividade inerente a esse tipo de cuidado. Pesquisa qualitativa com 39 enfermeiros psiquiátricos australianos revelou que embora eles reconheçam a importância da contenção mecânica para proteger $\mathrm{o}$ paciente e as demais pessoas em algumas situações de surto, não se sentem confortáveis em realizá-lo. ${ }^{16}$

Embora uma parte expressiva dos enfermeiros e dos pacientes tenha considerado a contenção mecânica menos importante, apenas um técnico de enfermagem teve essa mesma opinião. Acredita-se que os técnicos pensem de modo diferente sobre a contenção por estarem em contato direto com os pacientes e serem os principais alvos de suas eventuais agressões.

Os resultados deste estudo oferecem dados importantes para que os enfermeiros possam refletir sobre sua prática profissional. Mesmo que tenha sido realizado em um hospital específico, cada leitor pode estender essa reflexão para a sua própria prática assistencial: Será que o cuidado de enfermagem que eu ofereço é o que o paciente necessita? Acredita-se que a discrepância entre o que o paciente 
valoriza e o que ocorre na prática pode prejudicar a satisfação em relação à internação psiquiátrica e ao cuidado de enfermagem. Pretende-se, em outros estudos, investigar não apenas os cuidados mais valorizados e frequentes, mas a importância de cada um deles na perspectiva dos profissionais de enfermagem e dos pacientes. Embora o presente estudo investigue um assunto importante para a assistência de enfermagem psiquiátrica, a partir de uma técnica indireta para a coleta de dados com recurso projetivo (fotos), reconhece-se como limitação o número restrito de participantes.

\section{CONCLUSÃO}

$\mathrm{O}$ cuidado de enfermagem, na UPHG, ocorria de modo generalista com ênfase em cuidados técnicos básicos, uma vez que a administração de medicação e a verificação dos sinais vitais eram os cuidados mais frequentemente implementados, na opinião dos pacientes. $\mathrm{O}$ cuidado que os pacientes mais valorizam era o menos frequente, sugerindo que sua opinião não era considerada pela equipe de enfermagem da UPHG no planejamento da assistência. Enfermeiros, técnicos de enfermagem e pacientes tinham opiniões diferentes sobre os cuidados mais importantes, durante a internação psiquiátrica. Os enfermeiros valorizaram mais as "atividades burocráticas", os técnicos de enfermagem, "ouvir atentamente" e os pacientes, o "suporte espiritual”.

Conflitos de interesse: não há conflitos de interesse a declarar.

\section{REFERENCIAS}

1. Poirier J, Clarac F, Barbara JG, Broussolle E. Figures and institutions of the neurological sciences in Paris from 1800 to 1950 . Part IV: psychiatry and psychology. Rev Neurol. (Paris). [Internet]. 2012 [citado em 10 jul 2018]; 168(5):389-402. Disponível em: https://doi.org/10.1016/j.neurol.2012.02.00 7

2. Hein LC, Scharer KM. A modern history of psychiatric-mental health nursing. Arch Psychiatr Nurs. [Internet]. 2015 [citado em 04 jun 2018]; 29(1):49-55. Disponível em: https://doi.org/10.1016/j.apnu.2014.10.003 3. Dempsey A, Ribak J. The future of the psychiatric mental health clinical nurse specialist: evolution or extinction. Psychiatr Clin North Am. [Internet]. 2015 [citado em 01 jul 2018]; 38(1):121-130. Disponível em: https://doi.org/10.1016/j.cnur.2012.02.003 
4. Barker P. Psychiatric and mental health nursing: the craft of caring. London:

Hodder Arnold; 2017. 846p.

5. Townsend MC. Enfermagem psiquiátrica: conceitos de cuidados na prática baseada em evidências. Rio de Janeiro: Guanabara Koogan; 2014. 956p. 6. Oliveira RM, Siqueira Júnior AC, Furegato ARF. Perceptions on psychiatric nursing care at a general hospital inpatient unit. Acta Sci, Health Sci. [Internet]. 2016 [citado em 10 jul 2018]; 38(1):39-47. Disponível em: http://dx.doi.org/10.4025/actascihealthsci.v $38 \mathrm{i} 1.28673$

7. Loch AA. Stigma and higher rates of psychiatric re-hospitalization: São Paulo public mental health system. Rev Bras Psiquiatr. [Internet]. 2012 [citado em 10 jul 2018]; 34(2):185-192. Disponível em: http://www.scielo.br/scielo.php?script=sci _arttext\&pid=S1516-

$44462012000200011 \& \operatorname{lng}=\mathrm{en} \& \mathrm{nrm}=\mathrm{iso} \& \mathrm{t}$ $\operatorname{lng}=\mathrm{en}$

8. Jaramillo-Gonzalez LE, SanchezPedraza R, Herazo MI. The frequency of rehospitalization and associated factors in Colombian psychiatric patients. BMC Psychiatry. [Internet]. 2014 [citado em 12 mar 2018]; 14(161):1-7. Disponível em: https://doi.org/10.1186/1471-244X-14-161 9. Horta WA. Processo de enfermagem. São Paulo: Guanabara; 2011. (Enfermagem Essencial).

10. Bulechek GM, Butcher HK, Dochterman JM, Wagner CM. Nursing Interventions Classification (NIC). United States: Elsevier; 2016.

11. Timmins F, Murphy M, Neill F, Begley T, Sheaf G. An exploration of the extent of inclusion of spirituality and spiritual care concepts in core nursing textbooks. Nurse Educ Today. [Internet]. 2015 [citado em 26 jun 2018]; 35(1):277-282. Disponível em: https://doi.org/10.1016/j.nedt.2014.05.008
12. Lanfredi M, Candini V, Buizza

C, Ferrari C, Boero ME, Giobbio GM, et al. The effect of service satisfaction and spiritual well-being on the quality of life of patients with schizophrenia. Psychiatry Res. [Internet]. 2014 [citado em 08 jul 2018]; 216(2):185-191. Disponível em: https://doi.org/10.1016/j.psychres.2014.01. 045

13. Olson JK. Knowledge required to use the power of spirituality in healthcare. Acta Paul Enferm. [Internet]. 2015 [citado em 10 jul 2018]; 28(2):3-4. Disponível em: http://dx.doi.org/10.1590/19820194201500017.

14. Drori T, Guetta H, Ben Natan M, Polakevich Y. Effect of mental health nurses' beliefs and knowledge of medication on their use of strategies to improve medication adherence. Int $\mathbf{J}$ Mental Health Nurs. [Internet]. 2014 [citado em 01 jul 2018]; 23(4):374-380. Disponível em: https://doi.org/10.1111/inm.12062 15. Giordani JN, Bisogno SBC, Silva LAA. Perception of nurses regarding management activities for user assistance. Acta Paul Enferm. [Internet]. 2012 [citado em 10 jul 2018]; 25(4):511-516.

Disponível em:

http://www.scielo.br/pdf/ape/v25n4/en_05. pdf

16. Muir-Cochrane EC, Baird J, McCann TV. Nurses' experiences of restraint and seclusion use in short-stay acute old age psychiatry inpatient units: a qualitative study. J Psychiatr Ment Health Nurs. [Internet]. 2015 [citado em 01 jun 2018]; 22(2):109-115. Disponível em: https://doi.org/10.1111/jpm.12189

RECEBIDO: 08/08/2017

APROVADO: $16 / 03 / 2018$

PUBLICADO: 07/2018 